\title{
Evaluation of Mammary Cancer in 7,12-Dimethylbenz(a) anthracene-Induced Wister Rats by Asymmetrical Temperature Distribution Analysis Using Thermography: A Comparison with Serum CEA Levels and Histopathology
}

\author{
S. P. Angeline Kirubha, ${ }^{1}$ M. Anburajan, ${ }^{1}$ B. Venkataraman, ${ }^{2}$ \\ R. Akila, ${ }^{2}$ D. Sharath, ${ }^{3}$ and Baldev Raj ${ }^{4}$ \\ ${ }^{1}$ Biomedical Engineering Department, School of Bioengineering, Faculty of Engineering and Technology, SRM University, \\ Kanchipuram District, Tamil Nadu, Kattankulathur 603203, India \\ ${ }^{2}$ Radiological Safety and Environmental Group, Indira Gandhi Centre for Atomic Research (IGCAR), \\ Tamil Nadu, Kalpakkam 603 102, India \\ ${ }^{3}$ Quality Assurance Division, Indira Gandhi Centre for Atomic Research (IGCAR), Tamil Nadu, Kalpakkam 603 102, India \\ ${ }^{4}$ Indira Gandhi Centre for Atomic Research (IGCAR), Tamil Nadu, Kalpakkam 603 102, India
}

Correspondence should be addressed to S. P. Angeline Kirubha, kirubhaangeline@gmail.com

Received 16 March 2012; Revised 5 June 2012; Accepted 28 June 2012

Academic Editor: George E. Plopper

Copyright ( $) 2012$ S. P. Angeline Kirubha et al. This is an open access article distributed under the Creative Commons Attribution License, which permits unrestricted use, distribution, and reproduction in any medium, provided the original work is properly cited.

\begin{abstract}
Animal surface temperature profile captured using infrared camera is helpful for the assessment of physiological responses associated with the regulation of body temperature. Diagnosing breast cancer in early stage itself has a greater effect on the prognosis. In this work, asymmetrical temperature distribution analysis of chemical carcinogen 7,12-dimethyl benz(a)anthraceneinduced in the lower right flank region of Wistar rats $(n=6)$ was carried out to test the potential of thermography in diagnosing mammary cancer and tumor growth over a period of nine weeks in comparison with histopathology results as standard. Temperature difference between the tumor induced lower right and left side of flank region was significant (with $P$ value $<0.001$ ), whereas in the abdomen and shoulder there was no significant difference in temperature between right and left sides. Percentage of asymmetrical temperature difference in the tumor induced lower flank region was 0.5 to $2 \%$, whereas in the other regions it was $<0.5 \%$. Green pixel distribution in RGB color histogram was asymmetrical in the tumor induced lower flank region. Temperature reduction was observed in the tumor induced region after the seventh day of carcinogen induction. Asymmetrical thermogram analysis is the best method of diagnosing mammary cancer and for studying tumor development.
\end{abstract}

\section{Introduction}

Breast cancer is an uncontrolled growth of breast cells originating from breast tissue, most commonly from the inner lining of milk ducts or the lobules as a result of mutations in the genes responsible for regulating the growth of cells and keeping them healthy. Worldwide $23 \%$ (1.38 million) of the total new cancer cases and $14 \%(458,400)$ of the total cancer deaths occurred in 2008. The estimated number of new breast cancer cases has been raised from about 641,000 cases in 1980 to 1.6 million cases in 2010 and 625,000 deaths in 2010 [1]. In
India, the number of new breast cancer cases is about 100,000 per year and this is expected to rise to 250,000 new cases per year by 2015 [2]. In mammography, magnetic resonance imaging (MRI), ultrasonography and fine needle aspiration cytology (FNAC), diagnosis is based on the anatomical changes of the breast, which may take up to ten years for the tumor to grow to a sufficient size to be detectable. Regular screening mammograms and computed tomography (CT) itself are cancer carcinogens. Early stage diagnosis of breast cancer is very difficult using mammographic screening. Sometimes MRI fails to distinguish between cancer tissue 
and edema fluid and not diagnosing ductal carcinoma in situ (DCIS). Many calcifications cannot be diagnosed by sonography. Scintimammography (SMM), single photon emission computed tomography (SPECT), and positron emission tomography (PET) can be used only as adjunct breast cancer imaging tools. These modalities cannot replace invasive procedures, as they have poor sensitivity to detect tumor deposits less than $1 \mathrm{~cm} \mathrm{[3].} \mathrm{Rather} \mathrm{than} \mathrm{anatomical} \mathrm{changes,}$ in digital infrared imaging, diagnosis based on the principle of analyzing temperature variation associated with metabolic activity and vascular circulation changes in the affected area [4]. IR imaging is being widely used for diagnosis, to study the progress of treatment of many disorders mainly in the wide area of rheumatology [5], dermatology, orthopedics, and circulatory abnormalities [6]. Thermography was widely used in diagnosis, prognosis of several diseases, and to study progress of treatment with animal model. Capsaicin can be used as a potential therapeutic adjuvant which significantly enhances the heating effects of biological tissues during tumor hyperthermia. Increase in temperature induced by intraperitoneal injection of capsaicin was more obvious than the other two methods of locally smeared with and subcutaneous injection of capsaicin [7]. Dynamic thermal imaging has been used intraoperatively during neurosurgical interventions, including real-time assessment of cerebral vessel patency and cerebral perfusion, monitoring of flap perfusion, and determination of tumor margins during resectional surgery [8-10]. The interpretation of cancer in human was proven on scoring 5 IR signs by the analysis of ROC (receiver operating characteristic) curve and AUC (area under the ROC curve) by the univariate logistic regression model for each IR sign and an age-adjusted multivariate logistic regression model [11]. Leonardo S. Motta and et al. designed an automatic approach to eliminate human factors and guarantee repeatability and robustness of the results and by preventing errors caused by fatigue [12].

Thermographic imaging could detect temperature changes as small as $0.1^{\circ} \mathrm{C}$ decrease on the skin surface at an early stage of tumor and it was proven in xenografts (MDAMB231, MCF7) induced nude mice model [13]. In contrast, the temperature and thermal conductivity in the cancerous breast were higher than the normal breast in human due to angiogenesis and higher metabolic rate of cancerous cells [14-16]. 13762 MAT mammary adenocarcinoma animal models were used for examining the effects of subcutaneous tumor growth on skin temperature and whether angiogenesis-induced changes in vascular density plays a role in any surface temperature changes [17]. DMBA induced animal model closely mimics human breast cancer as it actively metabolites with a capacity for damaging the DNA molecule, the main event in carcinogenesis initiation [1820]. Selenium in serum and neoplastic tissue in breast cancer was correlated with serum CEA level to analyze tumor growth [21]. Serum CEA (carcinoembryonic antigen) levels were measured to check the clinical improvement and to study the antitumour activity of histone $\mathrm{H} 1$ [22]. In this work, asymmetrical temperature distribution of thermal images of DMBA induced Wister rats was analyzed and the results were compared with histopathology report as standard and also with serum CEA levels to study the progress of tumor growth.

\section{Methodology}

2.1. Animals. Female Wister rats $(n=8)$ weighing $300-350 \mathrm{~g}$ and aged between 12 to 13 weeks were obtained from Kings Institute of Preventive Medicine, Guindy, Chennai, India. All animal experimental protocols used in our study were approved by the SRM University Animal Experimentation Ethics Committee. The animals were caged individually and kept at constant environmental and nutritional conditions throughout the experimental period with room temperature $23 \pm 2^{\circ} \mathrm{C}$ and humidity (50-55\%) with $12 \mathrm{~h}$ light/12 $\mathrm{h}$ dark cycle and were fed a standard pellet diet and with water ad libitum. In group I $(n=6)$, chemical carcinogen DMBA was induced and the other group was control group II $(n=2)$.

2.2. Breast Cancer Induction by Chemical Carcinogen. $20 \mathrm{mg}$ of 7,12-dimethylbenz(a)anthracene (DMBA) purchased from Sigma Chemicals was mixed with $0.5 \mathrm{ml}$ sunflower oil and $0.5 \mathrm{ml}$ saline to induce breast cancer in each rat. A single dose injection of DMBA was given subcutaneously into the right flanks only in test group I. $0.5 \mathrm{ml}$ of sunflower oil and $0.5 \mathrm{ml}$ of saline alone injected in the left flanks control side in all six rats and group II animals (control) were injected with $0.5 \mathrm{ml}$ of sunflower oil and $0.5 \mathrm{ml}$ of saline in both right and left flank region.

2.3. Infrared Thermal Imaging of Animals. Infrared camera (FLIR T400, FLIR Systems, Boston, MA, USA) with wide temperature range of -4 to $2192^{\circ} \mathrm{F}\left(-20\right.$ to $\left.1700^{\circ} \mathrm{C}\right)$, FOV $25^{\circ} \times 19^{\circ}$, and thermal sensitivity of $0.1^{\circ} \mathrm{C}$ was used in this study. As a baseline anterior to posterior view of whole body, thermal images of all the rats were taken under standard conditions at constant distance of $(12 \mathrm{~cm})$. Then immediately after chemical carcinogen injection starting from the first day thermal images was taken every day over a period of nine weeks.

2.4. Asymmetrical Temperature Distribution Analysis and Color Histogram Analysis. When the images are relatively symmetrical, small asymmetries may indicate suspicious region. Based on this physiology, tumor region can be identified by the analysis of asymmetrical temperature distribution. For asymmetrical temperature distribution analysis, the whole animal thermal image was divided into six region of interests (ROI), with size approximately $2 \mathrm{~cm} \times 2 \mathrm{~cm}$, as lower flanks region:right flank and left flank; abdomen region:right abdomen and left abdomen: shoulder region;right shoulder and left shoulder. In all these six regions average skin temperature was measured using FLIR 1.2 software in the specified ROIs. RGB color histogram of all the ROIs of one chemical carcinogen tumor induced rat was plotted using MATLAB 7.0.1 software.

2.5. Biomarker CEA Test. $2 \mathrm{ml}$ of blood was collected from each rat and serum was separated. Carcinoembryonic antigen levels $(\mathrm{ngm} / \mathrm{ml})$ in the serum of experimental animals 
were estimated by the ELISA kit after the MC has developed to a palpable size in the ninth week.

2.6. Histopathological Study. Tumors were removed from sacrificed rats and immediately fixed in $10 \%$ formalin fixative for $24 \mathrm{~h}$. The tissues were then dehydrated in ascending series of alcohol, kept in 1:1 mixture of absolute alcohol and benzene, and then in benzene for $1 \mathrm{~h}$ each. Finally, tissue pieces were embedded in paraffin wax and 7 micron thick sections were cut and spread on glass slides, stained with hematoxylin and eosin, slides mounted in DPX, and viewed under light microscope and photographed.

2.7. Statistical Analysis. Mean and standard deviation of skin temperature of ROIs for all the rats in group I were calculated for every week. Student's $t$ test was performed using SPSS 10.0 software to analyze the difference in symmetrical temperature distribution between right and left regions. Serum CEA levels are also reported as mean \pm SD for $(n=$ 6) group I and group II $(n=2)$.

\section{Results}

3.1. Tumor Induced Lower Flank Region. Mean and standard deviation of temperatures for different regions were calculated every week and tabulated. Table 1 shows the skin temperature distribution comparisons between tumor induced lower right flank region and control lower left flank region. In the base line Image, that is, before the chemical carcinogen induction, calculated mean and SD values of skin temperature at right flank and left flank were $38.98 \pm$ 0.28 and $39 \pm 0.13$, respectively. Percentage of asymmetrical temperature difference between right and left flanks was $0.05 \%$ and there was no significant difference between (i.e., $P$ $=0.854$ ) right and left flank regions. It showed symmetrical temperature distribution in the flank region before chemical carcinogen induction. Immediately after chemical carcinogen induction, mean and SD values of skin temperature at right flank and left flank were $36.62 \pm 0.2$ and $36.8 \pm 0.24$, respectively, and asymmetrical temperature difference $0.49 \%$ was observed. But there was no significant difference between (i.e., $P=0.105$ ) right and left flank region. After the first week of induction, significant temperature difference $(<0.001)$ was observed clearly till ninth week which indicates the asymmetrical temperature distribution in the tumor induced lower flank region. Temperature difference about $\left(0.2\right.$ to $\left.0.6^{\circ} \mathrm{C}\right)$ was found in the tumor induced region between lower right flank and left flank region. Percentage of asymmetrical temperature variation observed was $0.5 \%$ to $2 \%$. Greater difference in temperature of $1.73 \%$ was observed in the ninth week.

3.2. Abdomen Region. Similarly temperature distributions in the right and left abdomen region were also compared. From Table 1, it was observed that there was no significant difference in temperature distribution between the right abdomen and left abdomen region, before chemical carcinogen induction $(P=0.43)$, immediately after carcinogen induction $(P=0.76)$ and also after the tumor induction in all the weeks throughout the study period. Percentage asymmetrical temperature difference was $<0.51 \%$ in all the weeks. The temperature difference was very less, that is, $<0.19^{\circ} \mathrm{C}$.

3.3. Shoulder Region. Temperature distributions in the right and left shoulder region were also compared and tabulated in Table 1.There was no significant difference in temperature distribution between the right shoulder and left shoulder region, before chemical carcinogen induction, that is, in the baseline thermal image $(P=0.56)$, immediately after carcinogen induction $(P=0.461)$ and also after the tumor induction in all the weeks throughout the study period. Percentage asymmetrical temperature difference was $<0.44 \%$ in all the weeks.

Average skin temperature of all the tumor induced groups calculated weekly was plotted separately in all the three regions (flank, abdomen, and shoulder region) to visualize right and left side temperature variations. From Figures 1(b) and 1(c), it was clear that in abdomen and shoulder region, right and left side mean temperature curves were overlapping, whereas in tumor induced flank region difference in temperature distribution was found (Figure 1(a)) between right and left sides in all the weeks.

Percentage of asymmetrical temperatures distribution over the period of study still ninth week of the entire region were plotted. From Figure 2, temperature variation between tumor induced lower right and left flank region was about 0.5 to $2 \%$, whereas the temperature variation was very less in the other region (abdomen and shoulder region) about less than $0.5 \%$ throughout the period of study. Which clearly indicates that asymmetrical temperature distribution got in tumor induced lower flank region where as in other abdomen and shoulder region it is symmetrical.

3.4. Tumor Development versus Temperature Variation. To see the progress of tumor development, the thermogram ion images were converted into medical images which assign different colors to different span of temperatures using FLIR software. Figure 3 represents infrared images generated from a single animal taken on different weeks after subcutaneous injection of DMBA carcinogen. The box in each image (approximately $2 \mathrm{~cm} \times 2 \mathrm{~cm}$ ) corresponds to the different region of split. Progress reduction in skin temperature was observed which can be identified by the changing of color from orange into red (Figures 3(a), 3(b), 3(c), and 3(d)) in the tumor induced lower right flank region where as in other regions symmetrically there was no color change.

3.5. Asymmetrical Color Histogram Analysis. The color image was separated into different components as red, green, and blue with different bins and the respective histogram was plotted using MATLAB program for all the six ROIs and compared symmetrically. Asymmetrical green component, that is, the green pixel distribution was between 100 and 150 (Figure 4(a)) in the lower right flank, region, while in the lower left flank region it was 175 to 230 (Figure 4(b)) in group I mammary cancer induced rat. Whereas histogram of other abdomen and shoulder region (Figures 4(c), 4(d), 4(e), and $4(\mathrm{f}))$ is symmetrical, that is, pixel density distribution 


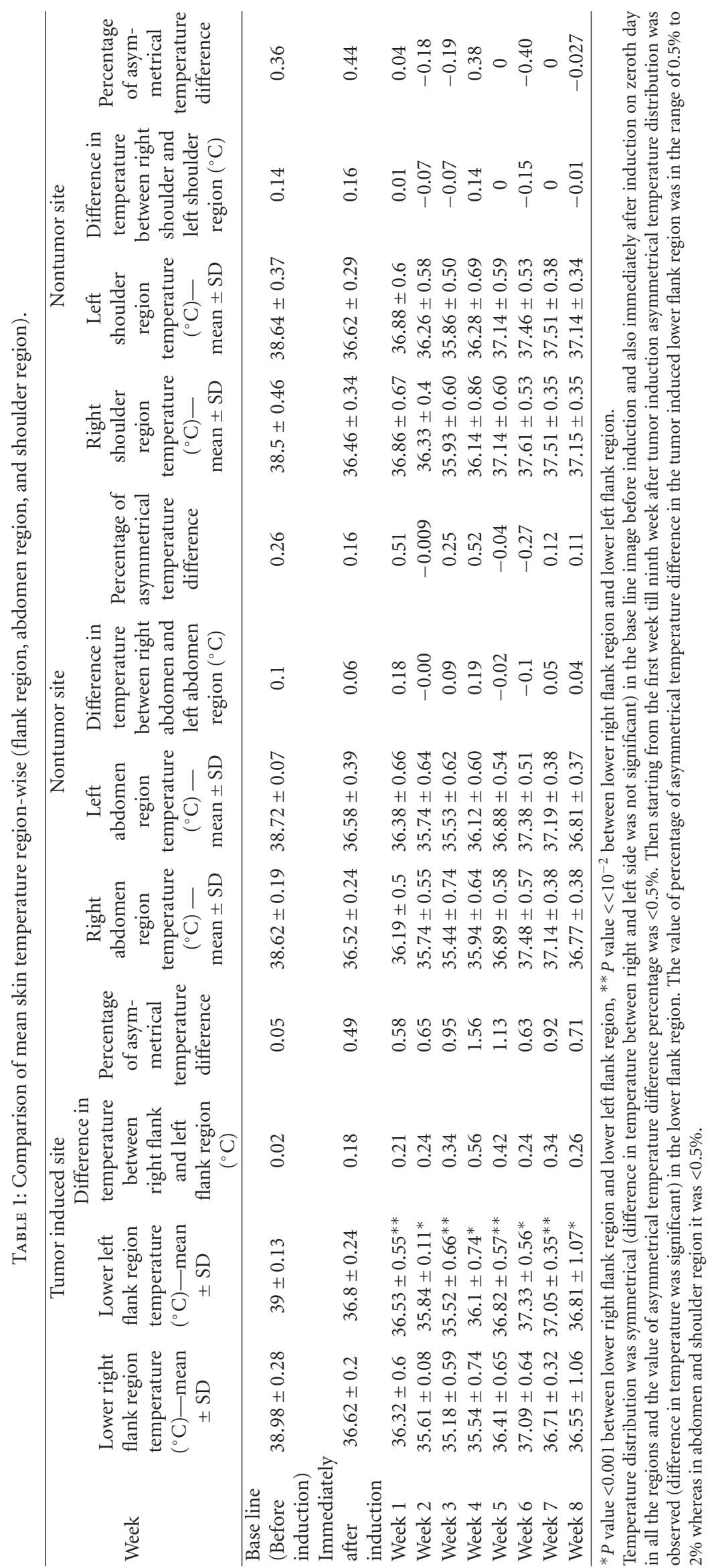




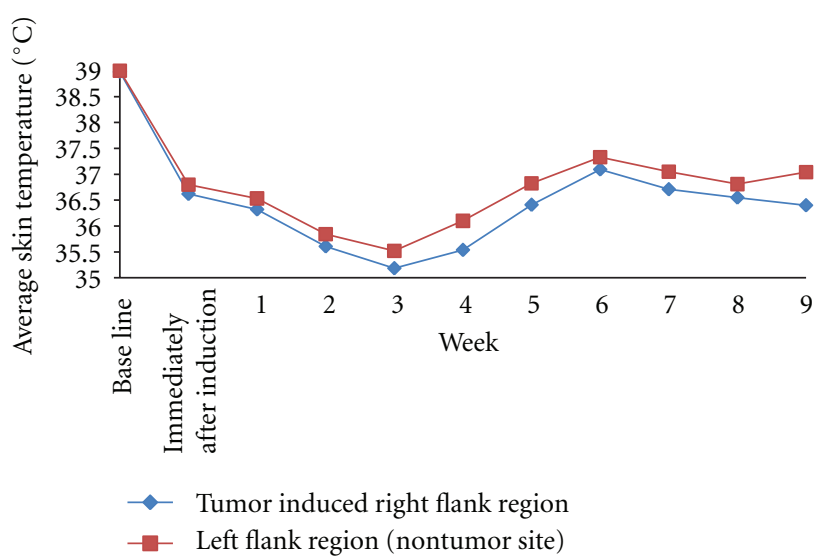

(a)

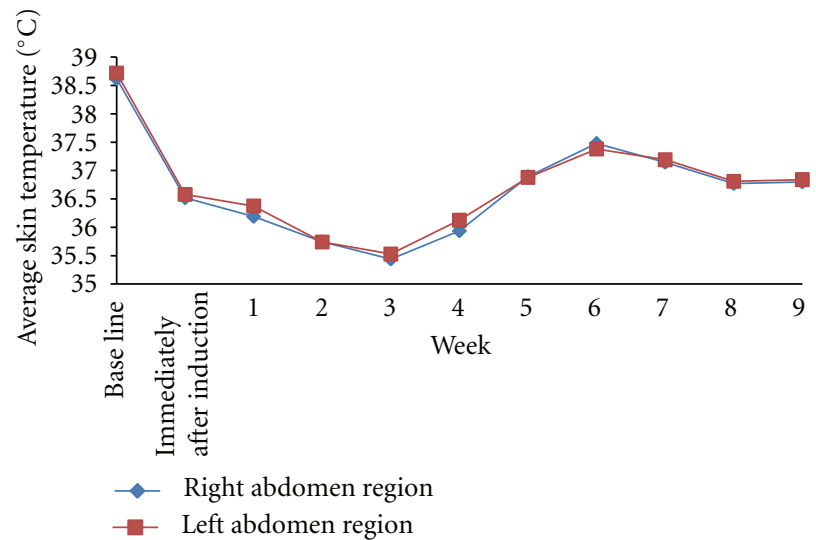

(b)

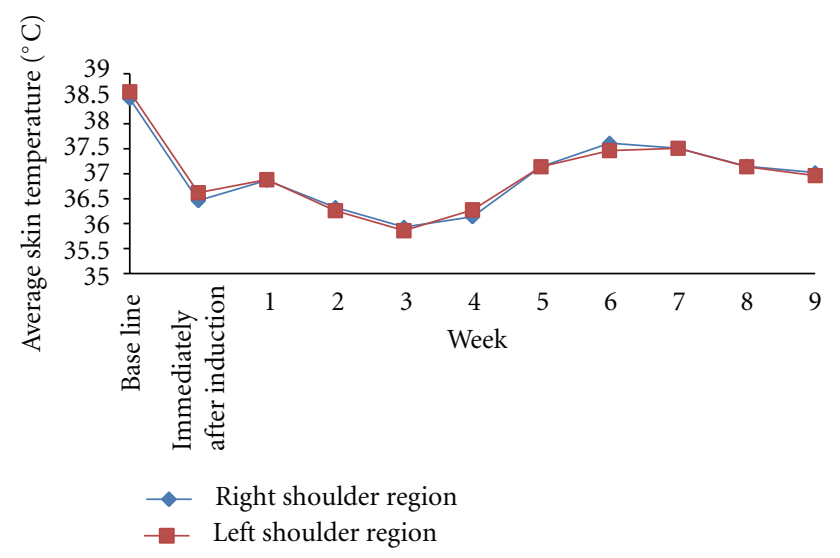

(c)

FIGURE 1: Average skin temperature in different regions (a) in the tumor induced lower flank region, (b) in the abdomen region, and (c) in the shoulder region. Mean \pm SD temperature values (week wise) for 6 tumor induced rats from the specific ROIs of right and left side for all regions (lower flank region, abdomen region, and shoulder region) were plotted. There was difference in temperature observed between right and left flank regions. Tumor induced right side (lower right flank side) temperature was less in all the weeks. There was no difference in temperature observed between right and left side of abdomen and shoulder regions.

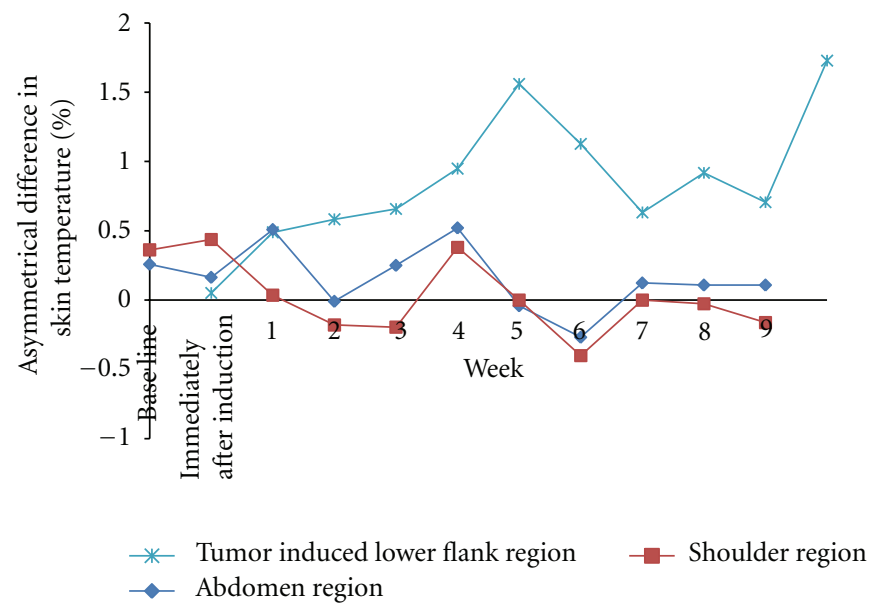

Figure 2: Percentage asymmetrical difference in skin temperature comparison between lower flank (tumor induced region), abdomen, and shoulder regions. The value of asymmetrical difference in skin temperature between left and right side ranges from 0.5 to $2.0 \%$ in the tumor induced flank region, whereas in the nontumor site (abdomen and shoulder regions) it was less than $0.5 \%$. 


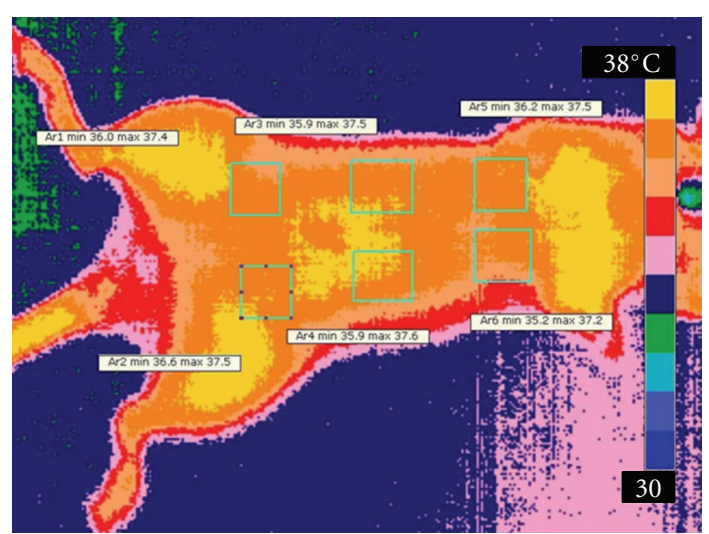

(a)

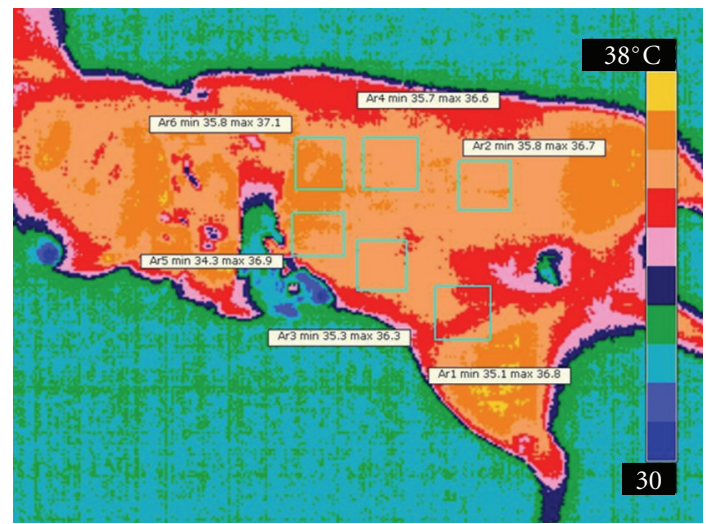

(c)

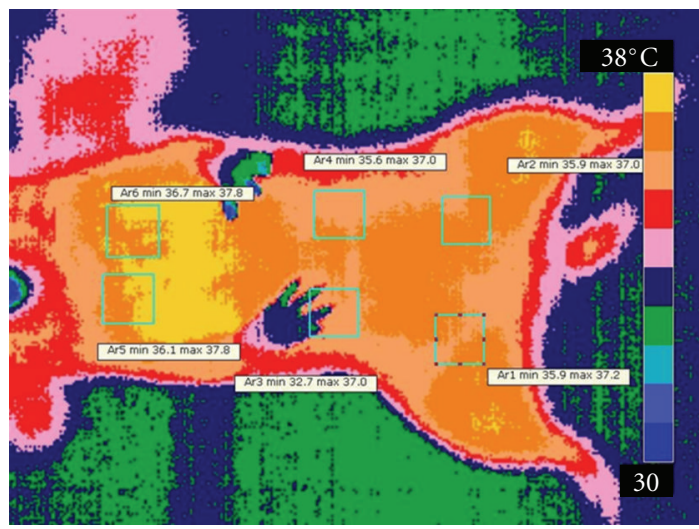

(b)

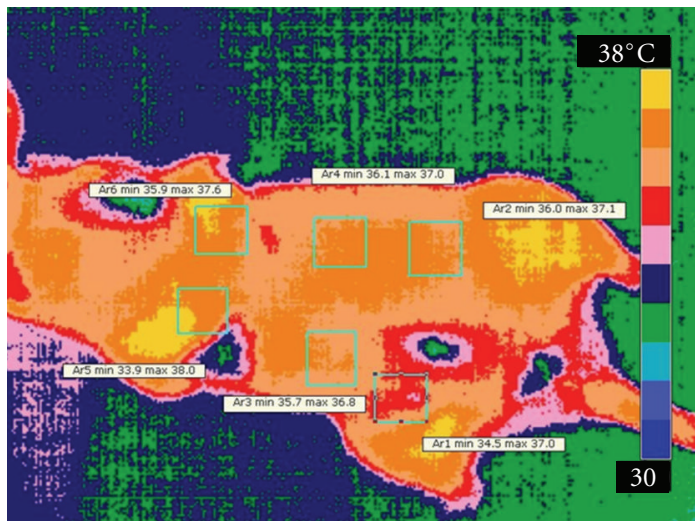

(d)

Figure 3: Whole body digital infrared images of a single rat taken on different weeks ((a) week 1, (b), week 2, (c) week 3, and (d) week 4) after tumor cancer induction by DMBA which show the tumor growth by color change from thick orange (a), then light orange (b), then red color(c), and then to red color with pink center in the fourth week indicating a reduction in skin temperature progressively.

was the same in right and left regions and also in group II control rat in all the regions (Figure 5).

In the RGB histogram analysis, asymmetry was observed only in the green components, whereas in red and blue components there was no predictable change.

3.6. CEA Results. Serum CEA level measured using ELISA kit also showed significant difference between mammary cancers induced group I and control group II. The mean tumor size was about $1.35 \mathrm{~cm}$ in group I and the value of CEA level was $0.426 \pm 0.05(\mathrm{ngm} / \mathrm{ml})$. In control group II CEA level was $0.186 \pm 0.01(\mathrm{ngm} / \mathrm{ml})$.

3.7. Histopathology Results. Histopathological results showed ductal carcinoma (grade III) in $(n=3)$ rats in chemical carcinogen induced group and grade IV metastases spread carcinoma in $n=3$ rats and mean tumor size was about $1.35 \mathrm{~cm}$. Figure 6(a) shows histopathology slides with different magnification of a rat with the infiltrating ductal carcinoma with size $1.5 \mathrm{~cm}$ (comedo carcinoma) showing several layers of neoplastic cells and central necrosis of single rat mammary tissue. Arrow indicating typical ductal cells arranged in tubular pattern. Single lymph nodal with metastasis was present. Tumor showed ductular and cribriform pattern more than 50\% ductal differentiation. Figure 6(b) shows microscopical section of another rat's mammary tissue which showed lobules of breast parenchyma with attached skeletal muscle. Single lymph node included in this section showed metastatic deposits.

\section{Discussion}

In this study, whole-body thermal images of the rats with induced tumor were obtained dynamically without anesthesia in order to rule out heat loss associated with anesthetic drugs. D. Colman et al. reported a significant amount of heat loss in an anesthetized Wister rat. The decrease in skin temperature, compared to the base line measurement after 30 minutes of induction, were found to be $1.75^{\circ} \mathrm{C}, 2.17^{\circ} \mathrm{C}$, and $1.9^{\circ} \mathrm{C}$ for the anesthetic drugs halothane, isofluorane, and sevoloflurane, respectively,[23]. In another study of 13762 MAT mammary adenocarcinoma induced rat model [17], it was observed that when the rat was anesthetized using ether, the measured skin temperature at the induced tumor site was lesser by $1^{\circ} \mathrm{C}$ than other non-tumor parts of rat.

In a xenograft induced breast tumor nude mice model using MDA-MB-231 and MCF7, the induced breast tumor had a lesser skin temperature on 6th day of tumor induction, 


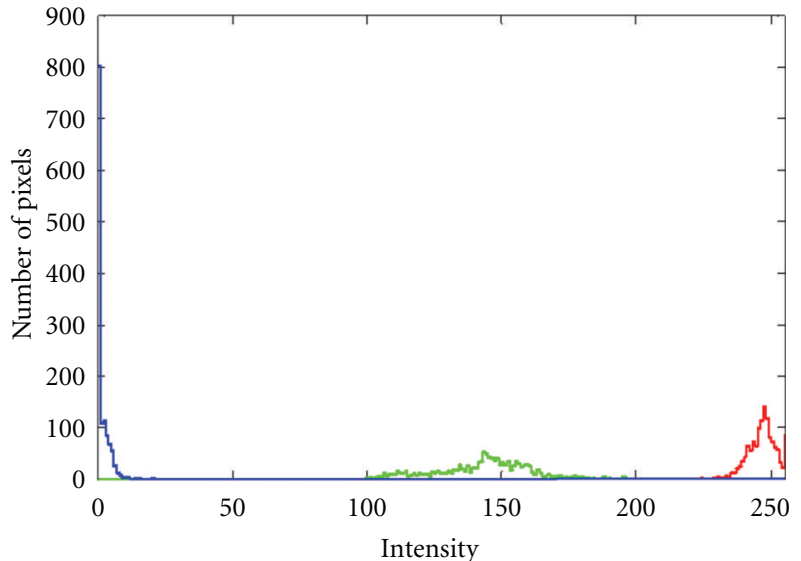

(a)

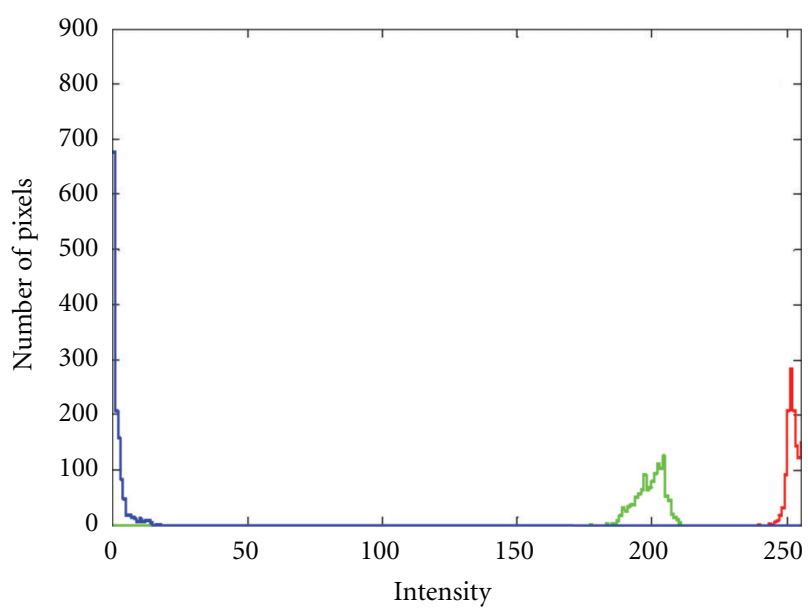

(c)

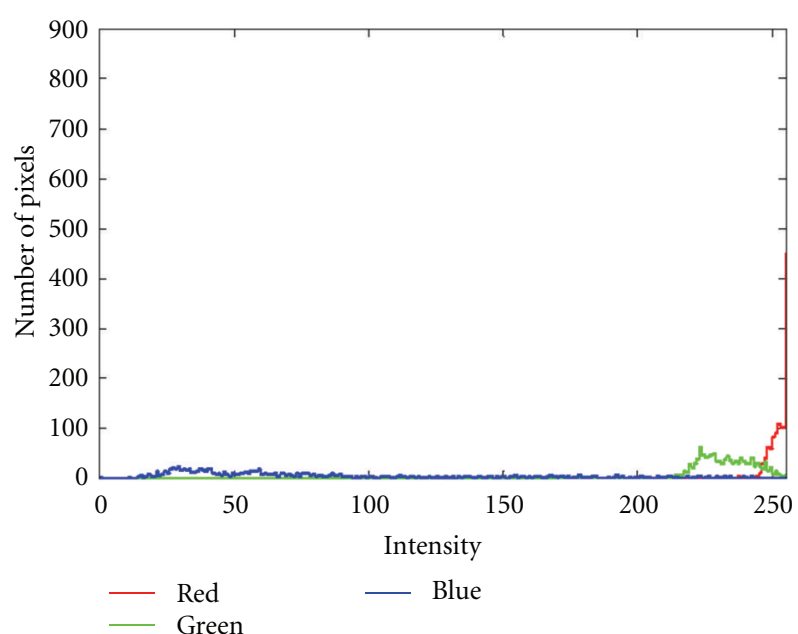

(e)

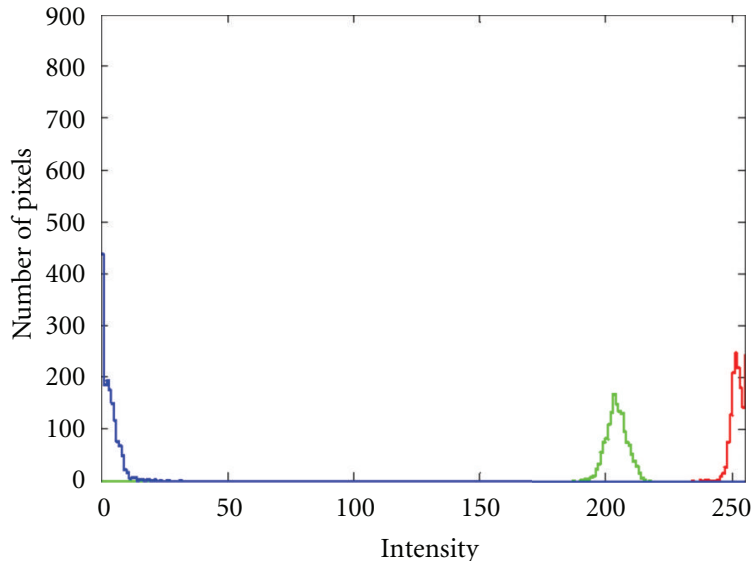

(b)

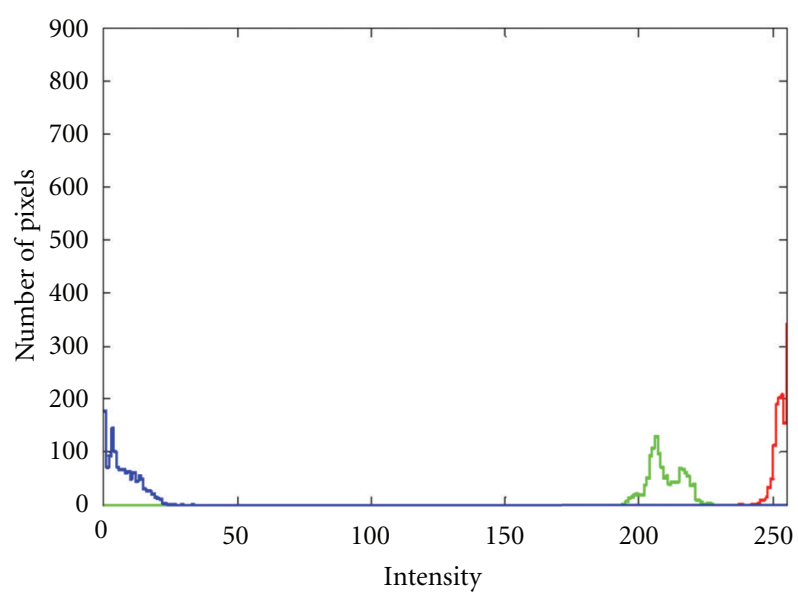

(d)

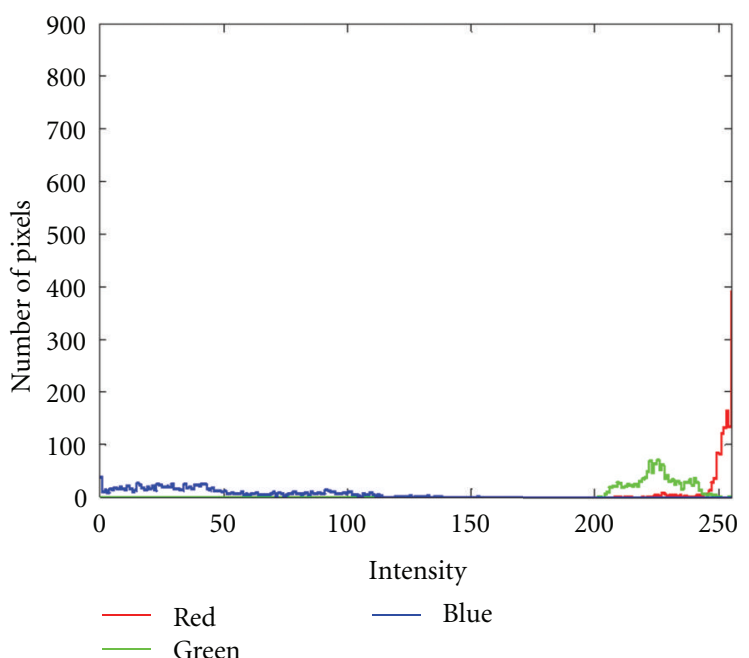

(f)

FIGURE 4: RGB histogram of single rat thermal image in different regions (a) lower right (tumor induced) flank region, (b) lower left flank region, (c) right abdomen region, (d) left abdomen region, (e) right shoulder region, and (f) left shoulder region. Red and blue pixel distributions were symmetrical between right and left sides in tumor induced flank region except green pixel distribution (asymmetrical). All red, green, and blue pixel distributions were symmetrical between corresponding right and left sides in nontumor sites (abdomen and shoulder regions). 


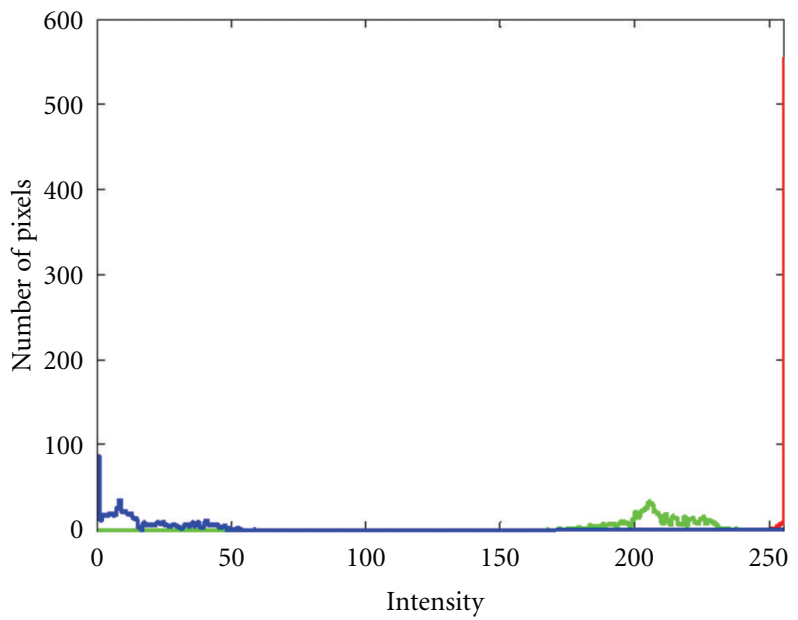

(a)

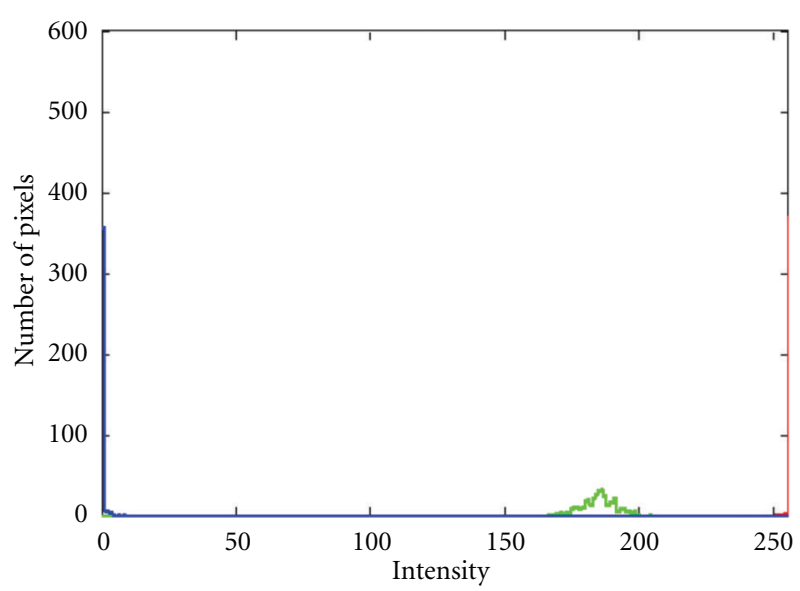

(c)

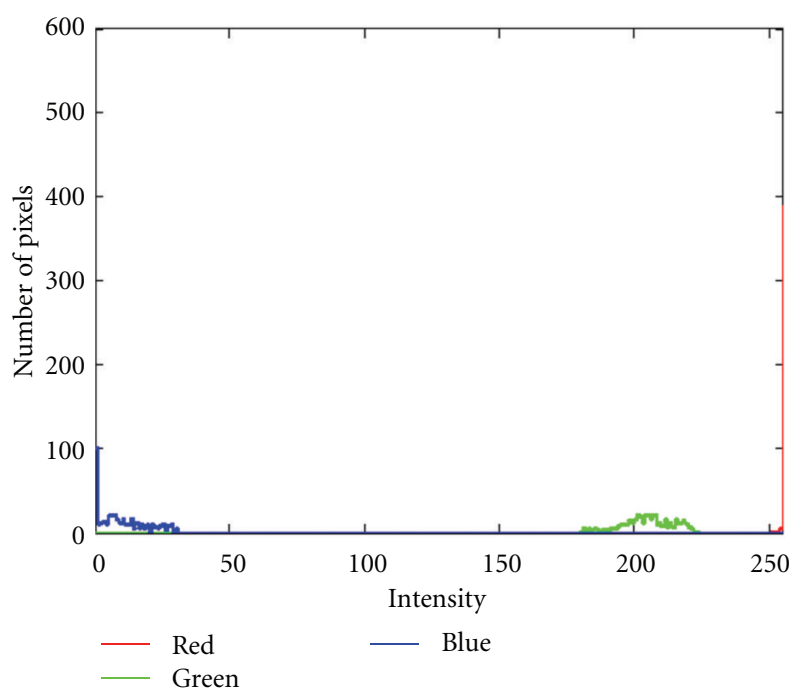

(e)

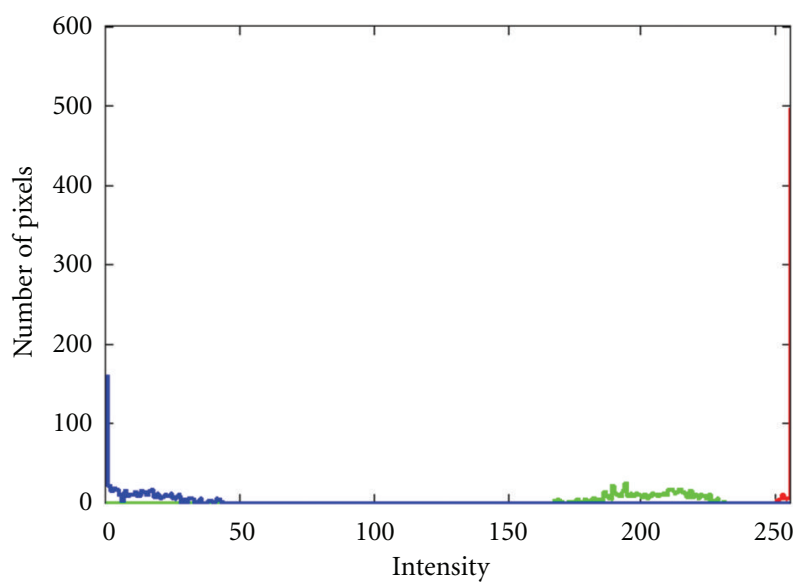

(b)

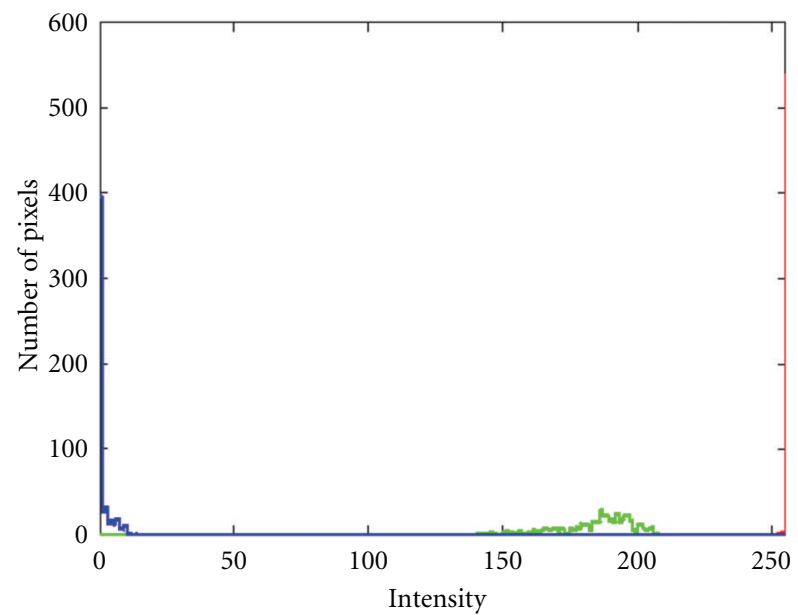

(d)

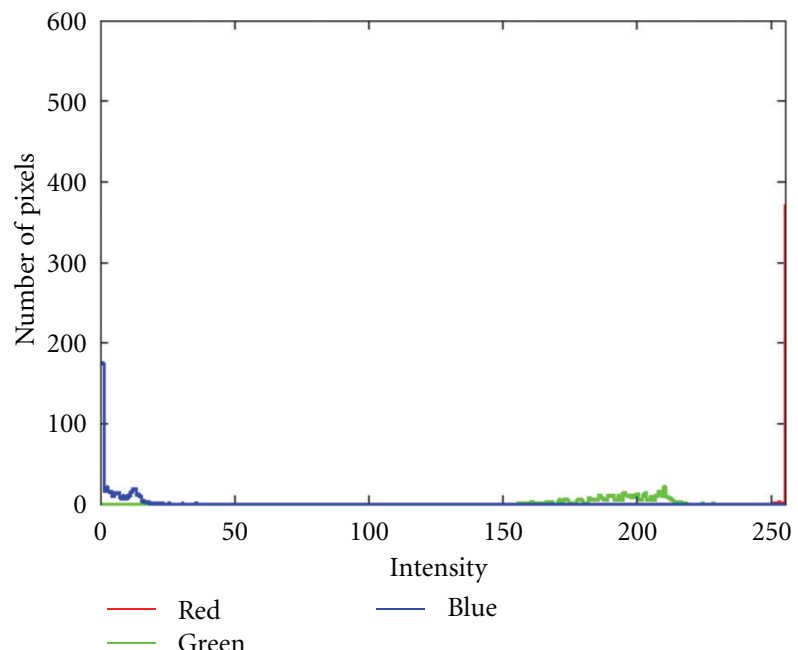

(f)

FIGURE 5: RGB histogram of single rat (control group II) thermal image in different regions (a) lower right flank region, (b) lower left flank region, (c) right abdomen region, (d) left abdomen region, (e) right shoulder region, and (f) left shoulder region. Red, blue, and green pixel distributions were symmetrical between right and left sides in all the regions (flank, abdomen, and shoulder). 

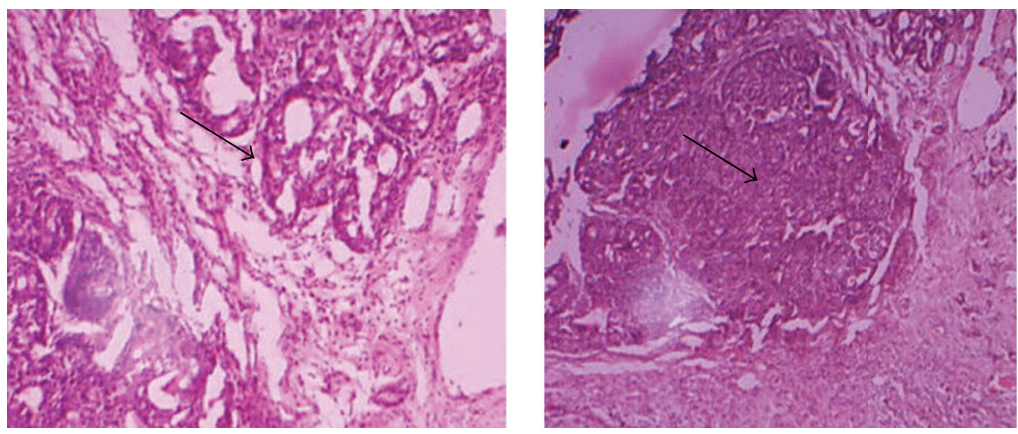

(a)
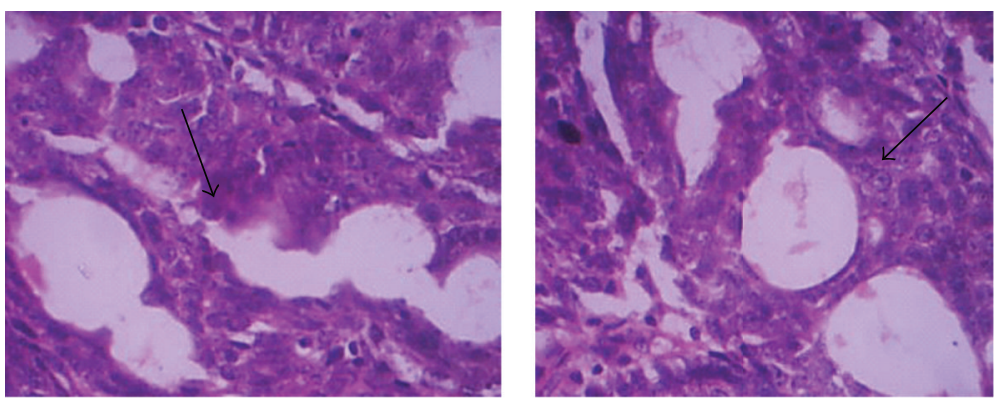

(b)
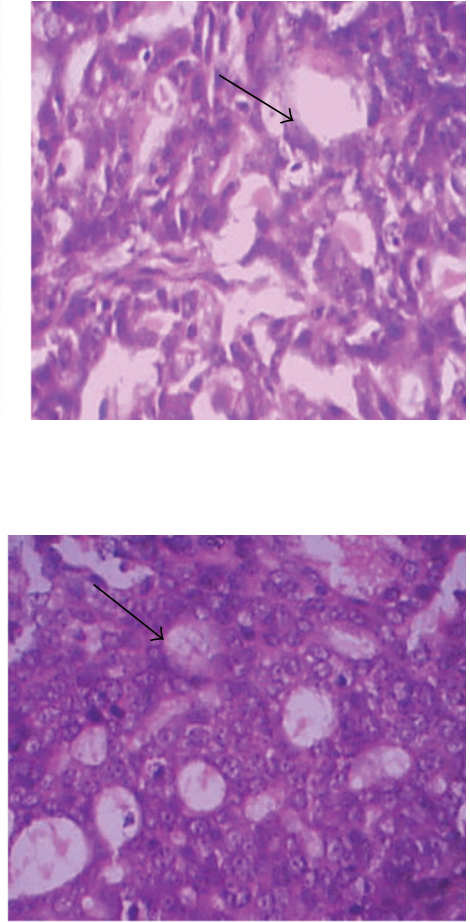

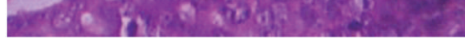

FIGURE 6: (a) Single rat with ductal carcinoma slides at different magnification. Arrow indicates ductal cells arranged in tubular pattern and tumor with 50\% ductal differentiation, (b) Single rat with metastasis stage carcinoma slides at different magnifications. Arrow indicates lymph node with metastasis deposits.

when comparing to nontumor parts of the mice. It was reported that the measured skin temperatures at the tumor site were lesser by $1.5^{\circ} \mathrm{C}$ and $3^{\circ} \mathrm{C}$ in MDA-MB-231 and MCF7 induced tumor mice models, respectively, [13]. In the present study, it was observed that right side of flank region, at which tumor was induced, had lesser skin temperature on 7th day of tumor induction, when comparing to base line measurements as well as other non-tumor parts of the rat. Further, during the third week after tumor induction, the measured mean skin temperature at the tumor site (right flank region) was lesser by $3.8^{\circ} \mathrm{C}$, when compared to non-tumor left flank region, which is agreed well with the xenograft induced mice models [13].

In a study of breast tumor in women using thermography, it was reported that the measured skin temperature at the tumor site was higher than the non-tumor part of the breast [24]. This finding was contradict with the findings of breast tumor rat models, in which the measured skin temperatures were lesser at the induced tumor site than its normal counterparts of the body $[13,17]$ and also in our study.

In the present study, the baseline measurements (before tumor induction) showed that the mean and SD value of skin temperature of whole-body rat was $38.98 \pm 0.28$. After tumor induction by chemical carcinogen, the measured mean and SD skin temperatures of the whole-body rat was found to be decreased progressively and it was $35.18 \pm 0.59$ during third week after tumor induction. The reason for reduction in mean whole-body skin temperature of the tumor bearing rat comparing to the baseline measurements may be due to the following: (i) poor functioning of newly developed blood vessels, and (ii) decreased blood flow at the tumor site by an autonomic nervous system [13, 17]. In the present study, the tumor induced right flank side of the rats showed a decrease in mean skin temperature values throughout the study period of nine weeks, when compared to the corresponding nontumor left flank side. The measured asymmetrical temperature difference at tumor bearing right flank side of the rats was ranged from 0.5 to $2.0 \%$, and it was statistically significant $(P<0.001)$. On the other hand, the non-tumor regions (abdomen and shoulder) of the rats showed a decrease in mean skin temperature values when compared to baseline measurements, but it was symmetrical in nature between right and left side of the rats and the measured skin temperature difference was less than $0.5 \%$.

Increase in temperature was observed from fourth week. This may be due to metastasis condition, that is, because of the spreading of cancer to other regions. This increase was because of angiogenesis and higher metabolic rate of welldeveloped cancerous cells.

From the first week to fourth week of the study, asymmetrical variation of skin temperature was visually observed (by noting changes in colors of the thermogram) in all the rats (Figures 3(a), 3(b), 3(c), and 3(d)). In the induced tumor 
site of the rat (right lower flank region), the skin temperature was found to be decreased progressively from first to fourth weeks of the study. It was observed visibly by noting the color changed from orange in the first week (Figure 3(a)) to light orange in the second week (Figure 3(b)), to red color in the third week (Figure 3(c)) and to red with center pink color in the fourth week (Figure 3(d)). On the other hand, in the non-tumor sites of the rat (abdomen and shoulder region), symmetrical nature of color variations were observed at right and left side of the rats. In human study, thermal images were analyzed for asymmetry between the patient's breasts based on different methods like Hurst coefficient [25], lacunarity coefficients [26], and by statistically texture analysis [27] to characterize the breast's texture.

thermal texture mapping (TTM) was found to be superior method for detection of malignant among the benign diseases of breast than mammography and ultrasound images [28]. In human breast tumor model, an increase in skin temperature in the tumor region was demonstrated by a shift in the number of pixels from blue color to red color that can be calculated by either the number of pixels or by the percentage of pixel distribution in the image. A healthy woman seems to be fairly balanced between the three primary colors, while the woman with breast tumor appears to have higher percentage of red color than blue color [29]. This higher percentage of red color may be an indication of rise in skin temperature; on the other hand, the lower percentage of green color may be an indication of decrease in skin temperature. Kapoor et al. [30] proposed an automatic pattern classification, which clearly diagnoses the pathological changes of the breasts based on pixel distribution asymmetries [30]. The red-green-blue (RGB) color histogram analysis of the thermal image is useful to study the asymmetric distribution of the skin temperature. In the tumor induced lower right flank region as well as in the non-tumor lower left flank region of the rat, red, and blue color pixel distributions were the same in the range of 230 to 255 and 0 to 10 , respectively, whereas the green color pixel distribution was shifted to a lower level of 100 to 150 at the tumor induced right flank than its corresponding value 175 to 230 measured at non-tumor left flank region of the rat. The shifting of green pixel distribution clearly indicates a decrease in skin temperature in the induced tumor site.

The non-tumor sites of abdomen and shoulder region of the rat showed symmetrical red, green, and blue pixel distributions between right and left sides of the rats. The measured red, green, and blue pixel distributions at abdomen region of the rat were found to be 240 to 255,175 to 220 , and 0 to 10 , respectively, and these values were the same in both right and left sides. Similarly, in the shoulder region of the rat, the measured red, green, and blue pixel distributions were found to be 240 to 255,200 to 240 , and 0 to 50 , respectively, which were the same in both right and left sides.

This study had few limitations. In this study, the number of rats used in this model was limited. Additional clinical and animal studies are required to prove whether thermography is useful in predicting the breast tumor well before. The finding of this study should be taken with the caution that it was related with small animal breast tumor model.

\section{Conclusion}

The findings of the study indicate that asymmetrical analysis of thermal images might have considerable potential in monitoring progress of tumor and also diagnosis of cancer in early stage itself. Significant $(<0.01)$ difference in asymmetrical skin temperature distribution was observed in tumor induced lower region, whereas in other regions temperature distribution was symmetrical. This was proven by asymmetrical pixel distribution in RGB histogram. And also green component of the infrared image plays an important role in the asymmetrical histogram analysis for diagnosing tumor in animal model with decrease in skin temperature. Further this study can be extended for studying the response to anticancer drugs.

\section{References}

[1] A. Jemal, F. Bray, M. M. Center, J. Ferlay, E. Ward, and D. Forman, "Global cancer statistics," CA Cancer Journal for Clinicians, vol. 61, no. 2, pp. 69-90, 2011.

[2] P. Yadav and D. P. Jaroli, "Breast cancer: awareness and risk factors in college-going younger age group women in Rajasthan," Asian Pacific Journal of Cancer Prevention, vol. 11, no. 2, pp. 319-322, 2010.

[3] S. VinithaSree, E. Yin-Kwee, R. Acharya, and O. Faust, "Breast imaging: a survey," World Journal of Clinical Oncology, vol. 2, no. 4, pp. 171-178, 2011.

[4] J. Kerr, "Review of the effectiveness of infrared thermal imaging (thermography) for population screening and diagnostic testing of breast cancer," NZHTA Tech Brief Series 2004, vol. 3 , no. 3, 2004.

[5] B. M. Sanchez, M. Lesch, D. Brammer, S. E. Bove, M. Thiel, and K. S. Kilgore, "Use of a portable thermal imaging unit as a rapid, quantitative method of evaluating inflammation and experimental arthritis," Journal of Pharmacological and Toxicological Methods, vol. 57, no. 3, pp. 169-175, 2008.

[6] L. Jiang, E. Y. K. Ng, A. Yeo et al., "A perspective on medical infrared imaging," Journal of Medical Engineering and Technology, vol. 29, no. 6, pp. 257-267, 2005.

[7] P. Zeng, Beijing, Z. Deng, and J. Liu, "Capsaicin-induced thermal enhancement on target tissues in hyperthermia," International Journal of Image, Graphics and Signal Processing, vol. 3, no. 3, pp. 49-55, 2011.

[8] M. Ashild and J. Mercer, "Intraoperative use of dynamic infrared thermography and indocyanine green fluorescence video angiography to predict partial skin flap loss," European Journal of Plastic Surgery, vol. 30, no. 6, pp. 269-276, 2008.

[9] R. D. Ecker, S. J. Goerss, F. B. Meyer, A. A. Cohen-Gadol, J. W. Britton, and J. A. Levine, "Vision of the future: initial experience with intraoperative real-time high-resolution dynamic infrared imaging," Journal of Neurosurgery, vol. 97, no. 6, pp. 1460-1471, 2002.

[10] A. M. Gorbach, J. D. Heiss, L. Kopylev, and E. H. Oldfield, "Intraoperative infrared imaging of brain tumors," Journal of Neurosurgery, vol. 101, no. 6, pp. 960-969, 2004.

[11] J. Wang, K. J. Chang, C. Y. Chen et al., "Evaluation of the diagnostic performance of infrared imaging of the breast: a preliminary study," BioMedical Engineering Online, vol. 9, article 3, 2010.

[12] L. Motta, A. Conci, R. Lima, and E. Diniz, Automatic Segmentation on Thermograms in Order to Aid Diagnosis and 2D 
Modeling, Anais CSBC, Belo Horizonte-Minas Gerais, Brazil, 2010.

[13] C. Song, V. Appleyard, K. Murray et al., "Thermographic assessment of tumor growth in mouse xenografts," International Journal of Cancer, vol. 121, no. 5, pp. 1055-1058, 2007.

[14] K. Lloyd Williams, F. J. Lloyd Williams, and R. S. Handley, "Infra-red thermometry in the diagnosis of breast disease," The Lancet, vol. 278, no. 7217, pp. 1378-1381, 1961.

[15] J. Head, F. Wang, C. Lipari, R. Elliott, and Keyserlingk, "The important role of infrared imaging in breast cancer," IEEE Engineering in Medicine and Biology Magazine, vol. 19, no. 3, pp. 52-57, 2000.

[16] J. Keyserlink, P. Ahlgren, E. Yu, N. Belliveau, and M. Yassa, "Functional infrared imaging of the breast," IEEE Engineering in Medicine and Biology Magazine, vol. 19, no. 3, pp. 30-41, 2000.

[17] W. Xie, P. McCahon, K. Jakobsen, and C. Parish, "Evaluation of the ability of digital infrared imaging to detect vascular changes in experimental animal tumours," International Journal of Cancer, vol. 108, no. 5, pp. 790-794, 2004.

[18] M. F. Dias, E. Sousa, S. Cabrita, J. Patrício, and C. F. Oliveira, "Chemoprevention of DMBA-induced mammary tumors in rats by a combined regimen of alpha-tocopherol, selenium, and ascorbic acid," Breast Journal, vol. 6, no. 1, pp. 14-19, 2000.

[19] L. Rajkumar, K. Balasubramanian, J. Arunakaran, P. Govindarajulu, and N. Srinivasan, "Influence of estradiol on mammary tumor collagen solubility in DMBA-induced rat mammary tumors," Cell Biology International, vol. 30, no. 2, pp. 164-168, 2006.

[20] J. Kaur, M. Sharma, P. Sharma, and M. Bansal, "Antitumor activity of lantadenes in DMBA/TPA induced skin Tumors in mice: expression of transcription factors," American Journal of Biomedical Sciences, vol. 2, no. 1, pp. 79-90, 2010.

[21] K. Charalabopoulos, A. Kotsalos, A. Batistatou et al., "Selenium in serum and neoplastic tissue in breast cancer: correlation with CEA," British Journal of Cancer, vol. 95, no. 6, pp. 674-676, 2006.

[22] G. Vani and C. S. Shyamala Devi, "Tumour suppressor activity of histone $\mathrm{H} 1$ in experimental mammary carcinoma," Indian Journal of Pharmacology, vol. 32, no. 3, pp. 210-213, 2000.

[23] D. Colman, M. C. B. F. De Melo, M. L. Brioschi, F. Silveira, and M. Cimbalista Júnior, "Analysis of heat loss using inhalation agents in rats subjected to laparotomy and increased intraabdominal pressure, using digital infrared thermal image," Revista Brasileira de Anestesiologia, vol. 52, no. 3, pp. 307-315, 2002.

[24] X. Tang, H. Ding, Y. E. Yuan, and Q. Wang, "Morphological measurement of localized temperature increase amplitudes in breast infrared thermograms and its clinical application," Biomedical Signal Processing and Control, vol. 3, no. 4, pp. 312$318,2008$.

[25] R. C. Serrano, A. Conci, M. Zamith, and R. C. F. Lima, "About the feasibility of Hurst coefficient in thermal images for early diagnosis of breast diseases," in Proceedings of the 11th PanAmerican Congress of Applied Mechanics (PACAM'10), Foz do Iguaçu, Brazil, January 2010.

[26] O. T. Da Silveira F, R. C. Serrano, A. Conci, R. H. C. De Melo, and R. C. F. Lima, "On using lacunarity for diagnosis of breast diseases considering thermal images," in Proceedings of the 16th International Conference on Systems, Signals and Image Processing (IWSSIP'09), pp. 1-4, Khalkis, Greece, June 2009.

[27] J. Koay, C. Herry, and M. Frize, "Analysis of breast thermography with an artificial neural network," in Proceedings of the 26th Annual International Conference of the IEEE Engineering in Medicine and Biology Society (EMBC'04), pp. 11591162, San Francisco, Calif, USA, September 2004.

[28] Y. Yuan, Q. Wang, Y. Tan et al., "Analysis of breast diseases examination with thermal texture mapping, mammography and ultrasound," in Proceedings of the 26th Annual International Conference of the IEEE Engineering in Medicine and Biology Society (EMBC’04), pp. 1166-1169, San Francisco, Calif, USA, September 2004.

[29] B. Morantz, Automated Analysis and Discrimination of Carcinoma in Situ of Thermographic (IR) Images, Imagery \& Technology Systems Division, Science Applications International, Tucson, Ariz, USA.

[30] P. Kapoor, S. Prasad, and E. Bhayana, "Real time intelligent thermal analysis approach for early diagnosis of breast cancer," International Journal of Computer Applications, vol. 1, no. 5, pp. 22-24, 2010. 


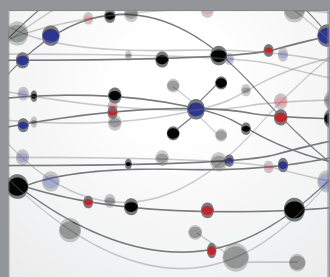

The Scientific World Journal
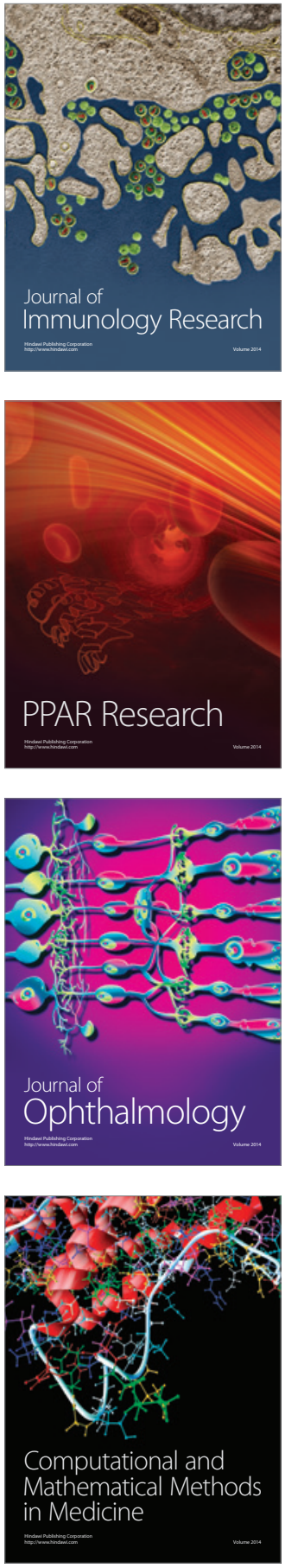

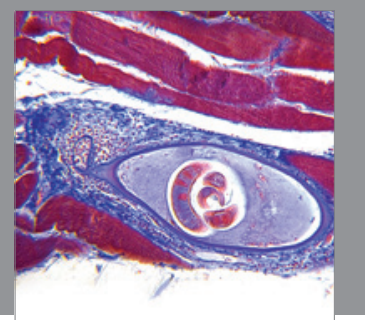

Gastroenterology

Research and Practice
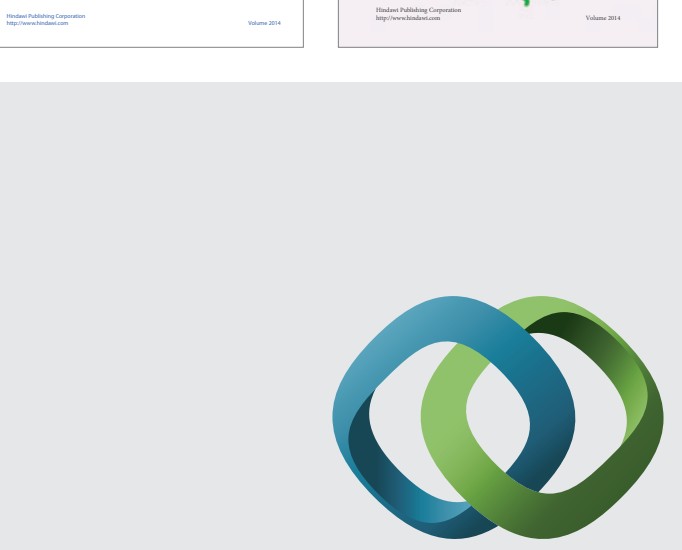

\section{Hindawi}

Submit your manuscripts at

http://www.hindawi.com
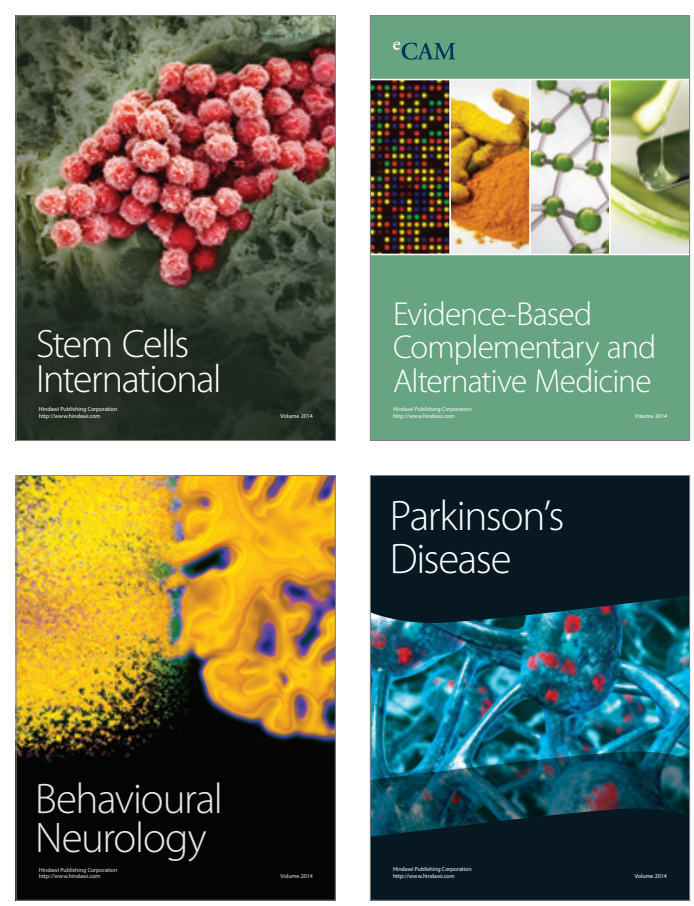

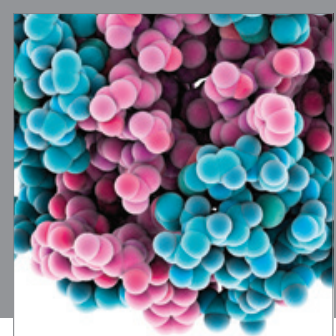

Journal of
Diabetes Research

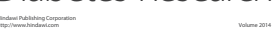

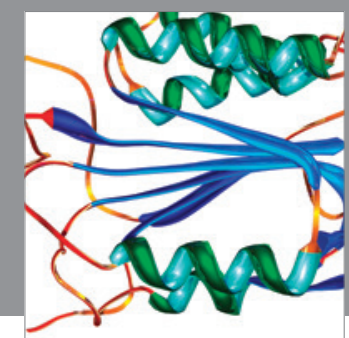

Disease Markers
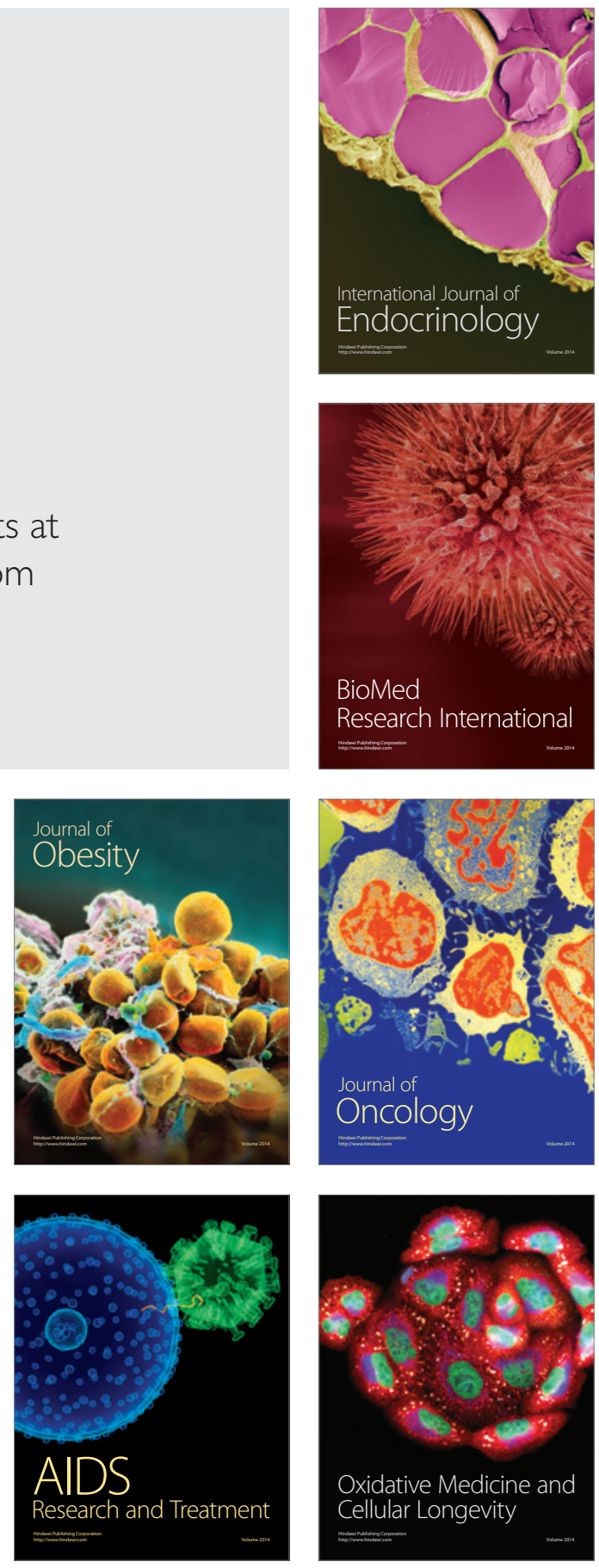